\title{
APPROXIMATE COMPLEMENTATION AND ITS APPLICATIONS IN STUDYING IDEALS OF BANACH ALGEBRAS
}

\author{
YONG ZHANG*
}

\begin{abstract}
We show that a subspace of a Banach space having the approximation property inherits this property if and only if it is approximately complemented in the space. For an amenable Banach algebra a closed left, right or two-sided ideal admits a bounded right, left or two-sided approximate identity if and only if it is bounded approximately complemented in the algebra. If an amenable Banach algebra has a symmetric diagonal, then a closed left (right) ideal $J$ has a right (resp. left) approximate identity $\left(p_{\alpha}\right)$ such that, for every compact subset $K$ of $J$, the net $\left(a \cdot p_{\alpha}\right)$ (resp. $\left(p_{\alpha} \cdot a\right)$ ) converges to $a$ uniformly for $a \in K$ if and only if $J$ is approximately complemented in the algebra.
\end{abstract}

\section{Introduction}

A subspace $E$ of a normed space $X$ is called approximately complemented in $X$ if there is a net $\left\{P_{\alpha}\right\}$ of continuous operators from $X$ into $E$ such that $P_{\alpha}(x)$ converges to $x$ uniformly on every compact subset of $E$. If in addition $\left\{P_{\alpha}\right\}$ can be chosen to be a bounded net, i.e., there is a constant $M$ such that $\left\|P_{\alpha}\right\| \leq M$ for all $\alpha$, then $E$ is called bounded approximately complemented in $X$. The notion of approximate complementation was first introduced in [17], where the author showed that if $E$ is approximately complemented in $X$ and if $\imath: E \rightarrow X$ is the inclusion mapping, then for every normed space $Y$, the mapping $\imath \otimes I_{Y}: E \hat{\otimes} Y \rightarrow X \hat{\otimes} Y$ specified by $\imath \otimes I_{Y}(a \otimes y)=\imath(a) \otimes y$, $a \in E, y \in Y$, is injective. Here $\hat{\otimes}$ denotes projective tensor product. Further investigation made in [19] suggests that the geometric property described in this notion may play an important role in studying ideals of Banach algebras.

It is a simple fact that if $E$ has the approximation property, then it is approximately complemented in every normed space $X$ that contains it. In light of this, one sees that Example 1.g.7 of [10] gives a Banach space that is not isomorphic to a Hilbert space and whose subspaces are all approximately complemented. It is also readily seen that in a Banach algebra $\mathfrak{A}$ a left ideal $I$ is approximately

\footnotetext{
* Research supported by NSERC.

Received January 1, 2002.
} 
complemented in $\mathfrak{U}$ if $I$ has a right approximate identity $\left(r_{\mu}\right)$ that satisfies the following condition, called condition (U): The net $\left(a \cdot r_{\mu}\right)$ converges to $a$ uniformly on every compact subset of $I$. Condition (U) for left and two-sided approximate identities can be defined similarly in an obvious way. A bounded left, right or two-sided approximate identity always satisfies condition (U). The Banach algebras constructed in [16, Section 3] do all have approximate identities satisfying condition (U), but none of them has a bounded approximate identity. A Banach algebra that has a bounded left approximate identity and a right approximate identity satisfying condition (U) but has no bounded right approximate identity is given in [4, Example 4.5]. Let $X$ be a Banach space and let $F(X)$ and $\mathscr{K}(X)$ be respectively the algebra of finite rank operators and the algebra of compact operators on $X$. Then $F(X)$ (resp. $\mathscr{K}(X)$ ) has a bounded left approximate identity if and only if the space $X$ has the bounded approximation property (resp. bounded compact approximation property) [5], while $F(X)($ resp. $\mathscr{K}(X))$ has a left approximate identity that satisfies condition (U) if and only if $X$ has the approximation property (resp. compact approximation property) [18]. Many examples reveal that, in dealing with ideals of Banach algebras, more often than not the existence of an approximate identity that satisfies condition $(\mathrm{U})$ can be asserted in terms of approximate complementation of the ideal. For instance, in a Segal algebra on a compact group, a closed right or left ideal has a left or right approximate identity satisfying condition (U) if and only if it is approximately complemented as a subspace in the Segal algebra [19].

In this note we first make a brief investigation of the problem when a subspace of a Banach space with the approximation property inherits the approximation property. Then we shall focus on ideals of amenable Banach algebras, discussing the existence of bounded and unbounded approximate identities in these ideals.

\section{The approximation property}

Recall that a Banach space $X$ has the approximation property if the identity operator on $X$ can be uniformly approximated on every compact subset of $X$ by finite rank operators on $X$. It is elementary that every Banach space with a basis has the approximation property. We know that in general the approximation property does not pass on to a closed subspace. Namely, a Banach space enjoying the approximation property may contain a closed subspace which lacks the approximation property [2], [3], [13]. However, except for several counterexamples, so far there is no result in the literature about conditions under which a subspace does inherit the approximation property. Using our approximate complementation we now can discuss this hereditability problem. 
Proposition 2.1. Suppose that $X$ is a Banach space having the approximation property. Then a subspace $E$ of $X$ has the approximation property if and only if it is approximately complemented in $X$.

Proof. It is readily seen that $E$ is approximately complemented in $X$ if it has the approximation property, since every continuous finite rank operator on $E$ can be extended to an operator from $X$ into $E$. For the converse we assume that $E$ is approximately complemented in $X$. Suppose that $K$ is a compact subset of $E$. Given $\varepsilon>0$, from the definition we can get a bounded operator $P_{1}: X \rightarrow E$, such that $\left\|P_{1}(k)-k\right\|<\varepsilon / 2$ for all $k \in K$. On the other hand, there is a bounded finite rank operator $P_{2}$ on $X$ such that $\left\|P_{2}(k)-k\right\|<\varepsilon /\left(2\left\|P_{1}\right\|\right)$ for all $k \in K$, due to the approximation property of $X$. Let $P=\left.\left(P_{1} \circ P_{2}\right)\right|_{E}$. Then $P$ is a bounded finite rank operator on $E$ and

$$
\|P(k)-k\| \leq\left\|P_{1}\left(P_{2}(k)-k\right)\right\|+\left\|P_{1}(k)-k\right\|<\varepsilon
$$

for all $k \in K$. Since $K$ and $\varepsilon>0$ are arbitrarily given, this implies that $E$ has the approximation property.

Consider the group algebra $L^{1}(G)$ of a locally compact group $G$. It is known that $L^{1}(G)$ always has the approximation property. On the other hand, it has been shown in [19] that for a compact group $G$ every closed two-sided ideal of $L^{1}(G)$ has an approximate identity satisfying condition $(\mathrm{U})$. As a consequence, every two-sided closed ideal in $L^{1}(G)$ with $G$ compact is approximately complemented in $L^{1}(G)$. Therefore we have shown the following:

COROLlary 2.2. Let $G$ be a compact group. Then every closed two-sided ideal of $L^{1}(G)$ has the approximation property.

It is inspiring to recall here the so-called Separable Extension Problem. The problem asks when a separable Banach space is complemented in every separable Banach space that contains it. This problem has been settled in Sobczyk-Zippin Theorem: An infinite dimensional separable Banach space is complemented in every separable Banach space containing it if and only if it is isomorphic to $c_{0}$ [12], [14], [20]. But $c_{0}$ is not complemented in $\ell_{\infty}[11,7.5]$. So finite dimensional Banach spaces are the only separable Banach spaces that are complemented in every containing Banach space. If we weaken the complementation requirement to approximate complementation, our Theorem 2.1 yields the following: A Banach space is approximately complemented in every Banach space that contains it if and only if it has the approximation property. 


\section{Ideals in amenable Banach algebras}

Amenability theory of Banach algebras was first introduced by B. E. Johnson in [7]. He showed later in [8] that a Banach algebra $\mathfrak{O}$ is amenable if and only if it has an approximate diagonal as defined in the following: A bounded net $\left(u_{v}\right) \subset \mathfrak{A} \hat{\otimes} \mathfrak{U}$ is an approximate diagonal for $\mathfrak{Q}$ if it satisfies $a u_{v}-u_{v} a \stackrel{\nu}{\rightarrow} 0$ and $\pi\left(u_{v}\right) a \stackrel{\nu}{\rightarrow} a$ for every $a \in \mathfrak{A}$, where $\hat{\otimes}$ denotes the projective tensor product and $\pi: \mathfrak{U} \hat{\otimes} \mathfrak{U} \rightarrow \mathfrak{U}$ is the product mapping defined by $\pi(a \otimes b)=a b$. An approximate diagonal $\left(u_{v}\right)$ for $\mathfrak{U}$ is said to be symmetric if it satisfies further the equality $a u_{v}=u_{v} a$ for all $v$ and all $a \in \mathfrak{A}$. Trivially, every contractible Banach algebra has a symmetric approximate diagonal. A simple example of infinite dimensional amenable Banach algebra with a symmetric approximate diagonal is $c_{0}$ consisting of all sequences of complex numbers converging to 0 , endowed with the uniform norm and the pointwise product. In fact, let $e_{i} \in c_{0}$ be given by $\left(e_{i}\right)_{j}=\delta_{i j}, i, j=1,2, \ldots$, and let $m_{n}=e_{1} \otimes e_{1}+e_{2} \otimes e_{2}+\cdots+e_{n} \otimes e_{n}$. Then $\left\{m_{n}\right\}$ is a symmetric approximate diagonal for $c_{0}$. Similar argument also shows that $\mathscr{K}(H)$, the algebra of compact operators on a Hilbert space $H$, has a symmetric approximate diagonal [6, VII.1.89]. Another example is the group algebra $L^{1}(G)$ with $G$ compact. We will discuss the details later in Proposition 3.4.

It is known that a closed left, right or two-sided ideal $J$ of an amenable Banach algebra $\mathfrak{A}$ has a bounded right, left or two-sided approximate identity if and only if it is weakly complemented in $\mathfrak{Y}$, i.e., $J^{\perp}=\left\{f \in \mathfrak{\mathfrak { V } ^ { * }}:\left.f\right|_{J}=0\right\}$ is complemented in the dual space $\mathfrak{A}^{*}[1]$. Our next theorem characterizes the same property in terms of bounded approximate complementation. Compared with the preceding known result, this theorem has the advantage that one does not need to refer to the dual space whose structure may be much more complicated. The relation between approximate complementation and weak complementation is unknown. The following example indicates that the former does not imply the latter.

Example 3.1. Consider the Banach space $L^{1}[0,1]$. It has a subspace isomorphic to $l_{2}$ [9, p. 57]. This subspace is approximately complemented in $L^{1}[0,1]$ since $l_{2}$ has the approximation property, but it is not weakly complemented in $L^{1}[0,1]$.

To see the last assertion, we assume, towards a contradiction, that this subspace were weakly complemented. Then $L^{\infty}[0,1]$ would have a complemented subspace isomorphic to $l_{2}^{*}=l_{2}$. Since $L^{\infty}[0,1]$ is isomorphic to $l_{\infty}[9$, p. 111], $l_{\infty}$ would have a complemented subspace which is isomorphic to $l_{2}$. But $l_{\infty}$ is a prime space $\left[9, \mathrm{p}\right.$. 57]. This would imply that $l_{\infty}$ were isomorphic to $l_{2}$, which is impossible. 
THEOREM 3.2. Suppose that $\mathfrak{A}$ is an amenable Banach algebra. Let $J$ be a closed left, right or two-sided ideal of $\mathfrak{Y}$. Then $J$ has a bounded right, left or two-sided approximate identity if and only if $J$ is bounded approximately complemented in $\mathfrak{A}$.

Proof. The necessity part is trivial. We prove the sufficiency for the case that $J$ is a closed left ideal of $\mathfrak{X}$. The proof for other cases is similar. Suppose that $J$ is bounded approximately complemented in $\mathfrak{A}$. Let $\left\{u_{v}\right\} \subset \mathfrak{A} \hat{\otimes} \mathfrak{U}$ be an approximate diagonal for $\mathfrak{A}$. Without loss of generality we may assume that

$$
u_{\nu}=\sum_{i=1}^{\infty} a_{i}^{(\nu)} \otimes b_{i}^{(\nu)}, \quad b_{i}^{(\nu)} \stackrel{i}{\rightarrow} 0 \quad \text { and } \quad \sum_{i=1}^{\infty}\left\|a_{i}^{(\nu)}\right\| \leq M<\infty .
$$

Let $P_{\alpha}: \mathfrak{U} \rightarrow J$ be a net of continuous operators satisfying $\left\|P_{\alpha}\right\| \leq K<\infty$ and $P_{\alpha}(a) \stackrel{\alpha}{\rightarrow} a$ uniformly on compact subsets of $J$. We define continuous linear mappings $\Phi_{\alpha}: \mathfrak{U} \hat{\otimes} \mathfrak{A} \rightarrow J$ by setting

$$
\Phi_{\alpha}(a \otimes b)=a P_{\alpha}(b) .
$$

Obviously, $\left\|\Phi_{\alpha}\right\| \leq\left\|P_{\alpha}\right\| \leq K$. Let

$$
\Gamma=\{(\varepsilon, \lambda): \varepsilon>0, \lambda \text { is a finite subset of } J\}
$$

with the partial order defined by $\left(\varepsilon_{1}, \lambda_{1}\right) \preccurlyeq\left(\varepsilon_{2}, \lambda_{2}\right)$ whenever $\varepsilon_{1} \geq \varepsilon_{2}$ and $\lambda_{1} \subseteq \lambda_{2}$. For each $(\varepsilon, \lambda) \in \Gamma$, chose $v=v(\varepsilon, \lambda)$ such that

$$
\left\|\pi\left(u_{\nu}\right) \ell-\ell\right\|=\left\|\sum_{i=1}^{\infty} a_{i}^{(\nu)} b_{i}^{(\nu)} \ell-\ell\right\|<\frac{\varepsilon}{4} \quad \text { and } \quad\left\|u_{\nu} \ell-\ell u_{\nu}\right\|<\frac{\varepsilon}{2 K}
$$

for all $\ell \in \lambda$. Fix this $\nu$. We then can take $\alpha=\alpha(\varepsilon, \lambda)$ such that

$$
\left\|P_{\alpha}\left(b_{i}^{(\nu)} \ell\right)-b_{i}^{(\nu)} \ell\right\| \leq \frac{\varepsilon}{4 M}
$$

for all $\ell \in \lambda$ and $i \geq 1$, since $\left\{b_{i}^{(v)} \ell: \ell \in \lambda, i \geq 1\right\}$ is a compact subset of $J$. Thus, for this choice of $v$ and $\alpha$, we have

$$
\left\|\Phi_{\alpha}\left(u_{\nu} \ell\right)-\ell\right\| \leq\left\|\sum_{i=1}^{\infty} a_{i}^{(\nu)}\left(P_{\alpha}\left(b_{i}^{(\nu)} \ell\right)-b_{i}^{(\nu)} \ell\right)\right\|+\left\|\sum_{i=1}^{\infty} a_{i}^{(\nu)} b_{i}^{(\nu)} \ell-\ell\right\|<\frac{\varepsilon}{2}
$$

for all $\ell \in \lambda$. On the other hand,

$$
\left\|\Phi_{\alpha}\left(\ell u_{v}-u_{v} \ell\right)\right\| \leq K\left\|u_{v} \ell-\ell u_{v}\right\|<\frac{\varepsilon}{2}
$$


for all $\ell \in \lambda$. Therefore,

$$
\left\|\ell \Phi_{\alpha}\left(u_{v}\right)-\ell\right\|=\left\|\Phi_{\alpha}\left(\ell u_{v}\right)-\ell\right\| \leq \varepsilon
$$

for all $\ell \in \lambda$. This shows that the net $\left(e_{(\varepsilon, \lambda)}\right)_{(\varepsilon, \lambda) \in \Gamma} \subset J$ with $e_{(\varepsilon, \lambda)}=$ $\Phi_{\alpha(\varepsilon, \lambda)}\left(u_{v(\varepsilon, \lambda)}\right)$ is a bounded right approximate identity for $J$.

If a Banach algebra has a symmetric approximate diagonal the method used in the above proof is also applicable for considering unbounded approximate identities in ideals.

Proposition 3.3. Let $\mathfrak{A}$ be an amenable Banach algebra that has a symmetric approximate diagonal. Let $J$ be a left (right) closed ideal of $\mathfrak{U}$. Then $J$ has a right (resp. left) approximate identity that satisfies condition $(U)$ if and only if $J$ is approximately complemented in $\mathfrak{A}$.

Proof. The proof is essentially the same as that of Theorem 3.2. One only needs to redefine the index set $\Gamma$ by letting

$$
\Gamma=\{(\varepsilon, \lambda): \varepsilon>0, \lambda \text { is a compact set of } J\}
$$

and to note that $\Phi_{\alpha}\left(\ell u_{v}\right)=\Phi_{\alpha}\left(u_{v} \ell\right)$ for all $\alpha$ and $v$ if $\left(u_{v}\right)$ is a symmetric approximate diagonal for $\mathfrak{A}$. We note that in this case the mappings $\Phi_{\alpha}$ are no longer uniformly bounded and hence the induced approximate identity $\left(e_{(\varepsilon, \lambda)}\right)$ for the ideal $J$ with $e_{(\varepsilon, \lambda)}=\Phi_{\alpha(\varepsilon, \lambda)}\left(u_{v(\varepsilon, \lambda)}\right)$ is no longer a bounded approximate identity.

As an application of the above proposition let us consider ideals in the group algebra $L^{1}(G)$ with $G$ compact. Our next result has been obtained in [19] by using a pure harmonic analysis method. Now proposition 3.3 provides another approach to the problem.

Proposition 3.4. Let $G$ be a compact group. Then a left (right) closed ideal of the group algebra $L^{1}(G)$ has a right (left) approximate identity satisfying condition $(U)$ if and only if it is approximately complemented in $L^{1}(G)$.

Proof. From Proposition 3.3 it suffices to show that $L^{1}(G)$ has a symmetric approximate diagonal. For a locally compact group $G$ it is well known that $L^{1}(G) \hat{\otimes} L^{1}(G)$ and $L^{1}(G \times G)$ are isometrically isomorphic as $L^{1}(G)$ bimodules. The isometric isomorphism is given by sending each elementary tensor $f \otimes g \in L^{1}(G) \hat{\otimes} L^{1}(G)$ to the function $f(s) g(t) \in L^{1}(G \times G)$. The $L^{1}(G)$-module actions on $L^{1}(G \times G)$ may be formulated as follows. For $s, t \in G$,

$$
(f \cdot u)(s, t)=\int_{G} f(\xi) u\left(\xi^{-1} s, t\right) d \xi, \quad(u \cdot f)(s, t)=\int_{G} u(s, t \xi) f\left(\xi^{-1}\right) d \xi
$$


where $f \in L^{1}(G), u \in L^{1}(G \times G)$. In the following we identify $L^{1}(G \times G)$ with $L^{1}(G) \hat{\otimes} L^{1}(G)$. Then the canonical product mapping $\pi$ is converted to $\Pi: L^{1}(G \times G) \rightarrow L^{1}(G)$ given by

$$
\Pi(u)(s)=\int_{G} u\left(\xi, \xi^{-1} s\right) d \xi, \quad s \in G, u \in L^{1}(G \times G) .
$$

Now define $\rho: L^{1}(G) \rightarrow L^{1}(G \times G)$ by

$$
\rho(f)(s, t)=f(s t), \quad s, t, \in G
$$

We have

$$
\rho(f * g)=f \cdot \rho(g)=\rho(f) \cdot g, \quad f, g \in L^{1}(G) .
$$

If $G$ is compact then $L^{1}(G)$ admits a bounded approximate identity $\left(e_{\alpha}\right)$ which lies in the center of $L^{1}(G)$. Let $u_{\alpha}=\rho\left(e_{\alpha}\right)$. Then a routine calculation shows that $\left(u_{\alpha}\right)$ is a symmetric approximate diagonal for $L^{1}(G)$.

It is worth mentioning that the amenability of $\mathfrak{U}$ is an irremovable condition in Proposition 3.3. In fact, G. A. Willis has shown in [15] that if $G$ is a non-amenable locally compact group (equivalently, if the group algebra $\mathfrak{U}=L^{1}(G)$ is not amenable) then no finite codimensional left ideal of $L^{1}(G)$ has a right approximate identity. With this remark the author wants to raise a question here to conclude this note: Whether the condition of the existence of a symmetric approximate diagonal is removable in Proposition 3.3? In particular, is Proposition 3.4 still valid if $G$ is merely an amenable group?

\section{REFERENCES}

1. Curtis Jr, P. C., and Loy, R. J., The structure of amenable Banach algebras, J. London Math. Soc. 40 (1989), 89-104.

2. Enflo, P., A counterexample to the approximation problem in Banach spaces, Acta Math. 130 (1973), 309-317.

3. Davie, A. M., The approximation problem for Banach spaces, Bull. London Math. Soc. 5 (1973), 261-266.

4. Dixon, P. G., Approximate identities in normed algebras, Proc. London Math. Soc. 26 (1973), 485-496.

5. Dixon, P. G., Left approximate identities in algebras of compact operators on Banach spaces, Proc. Roy. Soc. Edinburgh Sect. A 104 (1986), 169-175.

6. Helemskii, A. Ya., Banach and Locally Convex Algebras, Oxford University Press, Oxford, 1993.

7. Johnson, B. E., Cohomology in Banach Algebras, Mem. Amer. Math. Soc. 127, 1972.

8. Johnson, B. E., Approximate diagonals and cohomology of certain annihilator Banach algebras, Amer. J. Math. 94 (1972), 685-698. 
9. Lindenstrauss, J., and Tzafriri, L., Classical Banach Spaces I, Springer-Verlag, Berlin, Heidelberg, New York, 1977.

10. Lindenstrauss, J., and Tzafriri, L., Classical Banach Spaces II, Springer-Verlag, Berlin, Heidelberg, New York, 1979.

11. Phillips, R. S., On linear transformations, Trans. Amer. Math. Soc. 48 (1940), 516-541.

12. Sobczyk, A., Projection of the space $m$ on its subspace $c_{0}$, Bull. Amer. Math. Soc. 47 (1941), 938-947.

13. Szankowski, A., Subspaces without the approximation property, Israel J. Math. 30 (1978), 123-129.

14. Veech, W. A., Short proof of Sobczyk's Theorem, Proc. Amer. Math. Soc. 28 (1971), 627-628.

15. Willis, G. A., Approximate units in finite codimensional ideals of group algebras, J. London Math. Soc. 26 (1982), 143-154.

16. Willis, G. A., Examples of factorization without bounded approximate units, Proc. London Math. Soc. (3) 64 (1992), 602-624.

17. Zhang, Y., Nilpotent ideals in a class of Banach algebras, Proc. Amer. Math. Soc. 127 (1999), 3237-3242.

18. Zhang, Y., Unbounded approximate identities in algebras of compact operators on Banach spaces, Math. Proc. Cambridge Philos. Soc. 134 (2003), 187-192.

19. Zhang, Y., Approximate identities for ideals of Segal algebras on a compact group, J. Funct. Anal. 191 (2002), 123-135.

20. Zippin, M., The separable extension problem, Israel J. Math. 26 (1977), 373-387.

DEPARTMENT OF MATHEMATICS

UNIVERSITY OF MANITOBA

WINNIPEG, MANITOBA

R3T 2N2 CANADA

E-mail: zhangy@cc.umanitoba.ca 